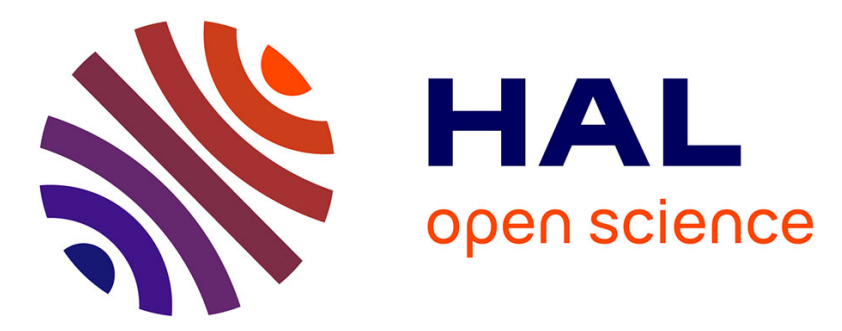

\title{
Time-periodic feedback stabilization for an ensemble of half-spin systems
}

Karine Beauchard, P.S. Pereira da Silva, Pierre Rouchon

\section{To cite this version:}

Karine Beauchard, P.S. Pereira da Silva, Pierre Rouchon. Time-periodic feedback stabilization for an ensemble of half-spin systems. 8th IFAC Symposium on Nonlinear Control Systems, Sep 2010, Bologna, Italy. hal-00577044

\section{HAL Id: hal-00577044 \\ https://hal-mines-paristech.archives-ouvertes.fr/hal-00577044}

Submitted on 16 Mar 2011

HAL is a multi-disciplinary open access archive for the deposit and dissemination of scientific research documents, whether they are published or not. The documents may come from teaching and research institutions in France or abroad, or from public or private research centers.
L'archive ouverte pluridisciplinaire HAL, est destinée au dépôt et à la diffusion de documents scientifiques de niveau recherche, publiés ou non, émanant des établissements d'enseignement et de recherche français ou étrangers, des laboratoires publics ou privés. 


\title{
Time-periodic feedback stabilization for an ensemble of half-spin systems
}

\author{
Karine Beauchard ${ }^{*}$ Paulo Sérgio Pereira da Silva ${ }^{* *, 2}$ \\ Pierre Rouchon ${ }^{* * *, 1}$ \\ * CMLA, ENS Cachan, CNRS, Universud, 61 Av. President Wilson, \\ F-94230 Cachan, FRANCE \\ Email: Karine.Beauchard@cmla.ens-cachan.fr \\ ** Escola Politécnica da USP - PTC Cep 05508-900 - São Paulo - SP \\ - BRAZIL Email : paulo@lac.usp.br, Fax: +(55)(11)30915718 \\ *** Mines Paris Tech, Centre Automatique et Systèmes, Unité \\ Mathématiques et Systèmes, 60 Bd Saint-Michel, 75272 Paris cedex \\ 06, FRANCE. Email: pierre.rouchon@mines-paristech.fr
}

\begin{abstract}
Feedback stabilization of an ensemble of non interacting half spins described by Bloch equations is considered. This system may be seen as a prototype for infinite dimensional systems with continuous spectrum. We propose an explicit feedback law that stabilizes asymptotically the system around a uniform state of spin $+1 / 2$ or $-1 / 2$. The closed-loop stability analysis is done locally around the equilibrium. The local convergence is shown to be a weak asymptotic convergence for the $H^{1}$ topology. The proof relies on an adaptation of the LaSalle invariance principle to infinite dimensional systems. Numerical simulations illustrate the efficiency of these feedback laws, even for initial conditions far from the equilibrium
\end{abstract}

Keywords: nonlinear systems, stabilization, quantum ensembles, Lyapunov stability, LaSalle invariance, infinite dimensional systems, continuous spectrum.

\section{INTRODUCTION}

\subsection{Infinite dimensional systems with continuous spectra}

Most controllability results available for infinite dimensional bilinear systems are related to systems with discrete spectra (see for instance, Beauchard and Coron [2006] for exact controllability results and Chambrion et al. [2009], Nersesyan [2010] for approximate controllability results). As far as we know, very few controllability studies consider systems admitting a continuous part in their spectra.

In Mirrahimi [2009] an approximate controllability result is given for a system with mixed discrete/continuous spectrum: the Schrödinger partial differential equation of a quantum particle in an $\mathrm{N}$-dimensional decaying potential is shown to be approximately controllable (in infinite time) to the ground bounded state when the initial state is a linear superposition of bounded states.

In Li and Khaneja [2006, 2009] a controllability notion, called ensemble controllability, is introduced and discussed for quantum systems described by a family of ordinary differential equations (Bloch equations) depending continuously on a finite number of scalar parameters and with a finite number of control inputs. Ensemble controllability means that it is possible to find open-loop controls that

\footnotetext{
$1 \mathrm{~KB}$ and PR were partially supported by the "Agence Nationale de la Recherche" (ANR), Projet Blanc C-QUID number BLAN-3139579 .

2 Partially supported by CNPq - Conselho Nacional de Desenvolvimento Cientifico e Tecnologico - Brazil, under grant 308465/2006-7.
}

compensate for the dispersion in these scalar parameters: the goal is to simultaneously steer a continuum of systems between states of interest with the same control input. Such continuous family of ordinary differential systems sharing the same control inputs can be seen as the prototype of infinite dimensional systems with purely continuous spectra.

The article Li and Khaneja [2009] highlights, the role of Lie algebras and non-commutativity in the design of a compensating control sequence and consequently in the characterization of ensemble controllability. In Beauchard et al. [2010], this analysis is completed by functional analysis methods developed for infinite dimensional systems governed by partial differential equations (see, e.g., Coron [2007] for samples of these methods). In Beauchard et al. [2010], several mathematical answers are given, with dicrimination between approximate and exact (simultaneous) controllability, and finite time and infinite time (simultaneous) controllability, for the Bloch equation.

The goal of this article is to investigate feedback stabilization of such specific infinite dimensional systems with continuous spectra. As in Mirrahimi [2009], the feedback design is based on a Lyapunov function closely related to the norm of the state space, a Banach space.

\subsection{The studied model}

We consider here an ensemble of non interacting half-spins in a static field $\left(\begin{array}{c}0 \\ 0 \\ B_{0}\end{array}\right)$ in $\mathbb{R}^{3}$, subject to a transverse radio 
frequency field $\left(\begin{array}{c}\tilde{v}(t) \\ -\tilde{u}(t) \\ 0\end{array}\right)$ in $\mathbb{R}^{3}$ (the control input). The ensemble of half-spins is described by the magnetization vector $M \in \mathbb{R}^{3}$ depending on time $t$ but also on the Larmor frequency $\omega=-\gamma B_{0}$ ( $\gamma$ is the gyromagnetic ratio). It obeys to the Bloch equation:

$$
\dot{M}(t, \omega)=\left(\tilde{u}(t) e_{1}+\tilde{v}(t) e_{2}+\omega e_{3}\right) \times M(t, \omega),
$$

where $-\infty<\omega_{*}<\omega^{*}<+\infty, \omega \in\left(\omega_{*}, \omega^{*}\right),\left(e_{1}, e_{2}, e_{3}\right)$ is the canonical basis of $\mathbb{R}^{3}, \times$ denotes the wedge product on $\mathbb{R}^{3}$ and $\dot{M}(t, \omega)=\frac{\partial M(t, \omega)}{\partial t}$. The equation (1) is a bilinear control system in which, at time $t$,

- the state is $(M(t, \omega))_{\omega \in\left(\omega_{*}, \omega^{*}\right)}$; for each $\omega, M(t, \omega) \in$ $\mathbb{S}^{2}$, the unit sphere of $\mathbb{R}^{3}$,

- the two control inputs $\tilde{u}(t)$ and $\tilde{v}(t)$ are real.

It must be stressed that $\tilde{u}(t)$ and $\tilde{v}(t)$ are common controls for all the members of the ensemble, and they cannot depend on $\omega$.

The state $M(t, \omega)=(x(t, \omega), y(t, \omega), z(t, \omega))$ of this ensemble of dynamic systems depends on $t$ and $\omega$ and its initial condition is a map $M^{0}:\left(\omega_{*}, \omega^{*}\right) \rightarrow \mathbb{S}^{2} \subset \mathbb{R}^{3}$, $M(0, \omega)=M^{0}(\omega)$. In coordinates, one may write

$$
\dot{x}=-\omega y+\tilde{v} z, \quad \dot{y}=\omega x-\tilde{u} z, \quad \dot{z}=-\tilde{v} x+\tilde{u} y .
$$

Formally, the spectrum of the operator $A$ defined by

$$
\left.A M\right|_{\omega}:=\omega e_{3} \times M(\omega)
$$

for every $M:\left(\omega_{*}, \omega^{*}\right) \rightarrow \mathbb{S}^{2}$, is $-\imath\left(\omega_{*}, \omega^{*}\right) \cup \imath\left(\omega_{*}, \omega^{*}\right)$ : for every $\omega_{0} \in\left(\omega_{*}, \omega^{*}\right)$, the eigenvector associated to $\pm \imath \omega_{0}$ is $\left(\begin{array}{c}1 \\ \mp \imath \\ 0\end{array}\right) \delta_{\omega_{0}}(\omega)$ where $\delta_{\omega_{0}}$ is the Dirac distribution in $\omega_{0}$ : $\int \phi(\omega) \delta_{\omega_{0}}(\omega) d \omega=\phi\left(\omega_{0}\right)$ for any continuous function $\phi$.

\subsection{Outline}

The goal of this article it to propose a first answer to the following question.

Local Stabilization Problem. Define an explicit control law $(\tilde{u}, \tilde{v})=(\tilde{u}(t, M), \tilde{v}(t, M))$ and a neighborhood $U$ of $-e_{3}$ (in some space of functions $\left(\omega_{*}, \omega^{*}\right) \rightarrow \mathbb{S}^{2}$ to be determined) such that, given any initial condition $M^{0} \in$ $U$, the solution of the closed loop system is defined for every $t \in[0,+\infty)$, is unique and converges to $-e_{3}$, when $t \rightarrow+\infty$, uniformly with respect to $\omega \in\left(\omega_{*}, \omega^{*}\right)$.

Section 2 is devoted to control design and closed-loop simulations: the feedback law is the sum of a Dirac comb and of a time-periodic feedback law based on a Lyapunov function; proposition 1 proved in appendix guaranties that the closed-loop initial value problem is always well defined; simulations illustrate the convergence rates observed for an initial state quite far from goal south pole $M \equiv-e_{3}$. In section 3 we state and prove the main convergence result, theorem 1: the closed-loop convergence towards the constant profile $M(\omega)=-e_{3}$ is shown to be local and weak for the $H^{1}$ topology on $M(\omega)$. Some concluding remarks are gathered in section 4 .

\section{LYAPUNOV $H^{1}$ APPROACH}

\subsection{The impulse-train control}

It is proved in Beauchard et al. [2010] that controls containing sums of Dirac masses are crucial to achieve the controllability of the Bloch equation. In view of the controls used in this reference, it is natural to consider a control that admits the following "impulse-train" structure

$$
\tilde{u}=u+\sum_{k=1}^{+\infty} \pi \delta(t-k T), \quad \tilde{v}=(-1)^{E\left(\frac{t}{T}\right)} v
$$

for some period $T>0(E(\gamma)$ denotes the integer part of the real number $\gamma$ ). The new controls $u$ and $v$ are bounded and measurable time functions. Then, after each impulse that is applied at time $t$ equal to $k T, x$ remains unchanged, but $y$ and $z$ are moved to their opposites (see $\mathrm{Li}$ and Khaneja [2009] for an explanation of this fact), that is $x\left(k T^{-}\right)=x\left(k T^{+}\right), \quad y\left(k T^{-}\right)=-y\left(k T^{+}\right), \quad z\left(k T^{-}\right)=-z\left(k T^{+}\right)$.

The resulting state diffeomorphism

$$
(x, y, z) \mapsto(\mathcal{X}=x, \mathcal{Y}=-y, \mathcal{Z}=-z)
$$

transforms (2) into

$$
\dot{\mathcal{X}}=\omega \mathcal{Y}-\tilde{v} \mathcal{Z}, \quad \dot{\mathcal{Y}}=-\omega \mathcal{X}-\tilde{u} \mathcal{Z}, \quad \dot{\mathcal{Z}}=\tilde{v} \mathcal{X}+\tilde{u} \mathcal{Y} .
$$

Let $\varsigma=(-1)^{E\left(\frac{t}{T}\right)}$. Considering the identification (4), one gets the following dynamics

$$
\dot{x}=-\varsigma \omega y+v z, \quad \dot{y}=\varsigma \omega x-u z, \quad \dot{z}=-v x+u y,
$$

with the new control $(u, v)$. It is as if, between $[k T,(k+$ 1)T] and $[(k+1) T,(k+2) T]$, one is changing the sign of $\omega$, but the solution, after the identification (4), remains continuous (but not differentiable in time at the instants $t=k T, k \in \mathbb{N}$ ). In other words, the application of the impulses at $t=k T$ changes the sense of rotation of the null input solution. One would expect that this impulse-train control is reducing the average dispersion of the solution. Roughly speaking, the dispersion observed for the openloop system (2) with $(\tilde{u}, \tilde{v})$ as input is strongly reduced and almost canceled for the open-loop system (5) with $(u, v)$ as input.

\subsection{Heuristics of the Lyapunov-like control}

Now let $Z(t, \omega)$ and $\Omega(t)$ be two complex numbers defined by

$$
Z=x+\imath y, \quad \Omega=v-\imath u
$$

where $x, y, z$ refer to the transformed dynamics (5). Then one may write (5) in the form

$$
\left\{\begin{aligned}
\dot{Z}(t, \omega) & =\imath \varsigma(t) \omega Z(t, \omega)+\Omega(t) z(t, \omega), \\
\dot{z}(t, \omega) & =-\Re[\Omega(t) \overline{Z(t, \omega)}]
\end{aligned}\right.
$$

where $\Re(\xi)$ (resp. $\bar{\xi}$ ) denotes the real part (resp. the complex conjugate) of a complex number $\xi \in \mathbb{C}$. It is easy to see that the following transformation

$$
\tilde{Z}(t, \omega)=Z(t, \omega) e^{-\imath \omega \int_{0}^{t} \varsigma(\tau) d \tau}
$$

converts the system into the driftless form

$$
\left\{\begin{aligned}
\dot{Z}(t, \omega) & =\Omega(t) z(t, \omega) e^{-\imath \omega \int_{0}^{t} \varsigma} \\
\dot{z}(t, \omega) & =-\Re\left[\Omega(t) \overline{Z(t, \omega)} e^{-\imath \omega \int_{0}^{t} \varsigma}\right]
\end{aligned}\right.
$$

where, for notation simplicity, one lets $Z(t, \omega)$ stand for $\tilde{Z}(t, \omega)$, and one lets $\int_{0}^{t} \varsigma$ stand for $\int_{0}^{t} \varsigma(\tau) d \tau$. 
For the moment one shall assume that the input $\Omega(t)$ will be chosen in such a way that the solution $(Z(t, \omega), z(t, \omega))$ of (6) does exist, it is unique and it is regular enough in a way that one may consider that the derivatives $Z^{\prime}(t, \omega)=$ $\frac{\partial Z}{\partial \omega}(t, \omega)$ and $z^{\prime}(t, \omega)=\frac{\partial z}{\partial \omega}(t, \omega)$ exists almost everywhere, and they are solutions of the differential equation ${ }^{3}$ that is obtained by differentiation of (6) with respect to $\omega$, namely

$$
\left\{\begin{array}{l}
\dot{Z}^{\prime}=\Omega\left\{\left[z^{\prime}-\imath\left(\int_{0}^{t} \varsigma\right) z\right] e^{-\imath \omega \int_{0}^{t} \varsigma}\right\}, \\
\dot{z}^{\prime}=-\Re\left\{\Omega\left[\bar{Z}^{\prime}-\imath\left(\int_{0}^{t} \varsigma\right) \bar{Z}\right] e^{-\imath \omega \int_{0}^{t} \varsigma}\right\},
\end{array}\right.
$$

where $\dot{Z}^{\prime}$ stands for $\frac{\partial}{\partial t} Z^{\prime}$, and $\dot{z}^{\prime}$ stands for $\frac{\partial}{\partial t} z^{\prime}$.

Now consider the following Lyapunov-like functional:

$$
\mathcal{L}=\frac{1}{2} \int_{\omega_{*}}^{\omega^{*}}\left\{G\left(\left|Z^{\prime}\right|^{2}+\left(z^{\prime}\right)^{2}\right)+|Z|^{2}\right\} d \omega
$$

where $G$ is a positive real number and $Z(t, \omega), Z^{\prime}(t, \omega)$ and $z^{\prime}(t, \omega)$ refer to the solutions respectively of (6) and (7). One may write

$$
\frac{d}{d t} \mathcal{L}(t)=\Re\left(\int_{\omega_{*}}^{\omega^{*}}\left\{G\left(\bar{Z}^{\prime} \dot{Z}^{\prime}+z^{\prime} \dot{z}^{\prime}\right)+\bar{Z} \dot{Z}\right\} d \omega\right)
$$

and so, taking into account (6) and (7), the fact that $\Omega(t)$ does not depend on $\omega$, one gets

$$
\frac{d}{d t} \mathcal{L}(t)=\Re[\Omega(t) H(t)]
$$

where

$$
H(t)=\int_{\omega_{*}}^{\omega^{*}}\left\{-\imath G\left(\int_{0}^{t} \varsigma\right)\left(\bar{Z}^{\prime} z-\bar{Z} z^{\prime}\right)+\bar{Z} z\right\} e^{-\imath \omega \int_{0}^{t} \varsigma} d \omega .
$$

Hence one may take $\Omega(t)=-K_{p} \bar{H}(t)$, where $K_{p}$ is a positive real number, obtaining

$$
\Omega(t)=-K_{p} \int_{\omega_{*}}^{\omega^{*}}\left\{\imath G\left(\int_{0}^{t} \varsigma\right)\left(Z^{\prime} z-Z z^{\prime}\right)+Z z\right\} e^{\imath \omega \int_{0}^{t} \varsigma} d \omega .
$$

It follows that $\frac{d}{d t} \mathcal{L}(t)=-\frac{1}{K_{p}}|\Omega(t)|^{2}$. Recall that $M=$ $(\Re(Z), \Im(Z), z)=(x, y, z)$. Consider the system (6) in closed loop with the control law (11), thereby called by closed loop system. The state space of this system is $H^{1}\left(\left(\omega_{*}, \omega^{*}\right), \mathbb{R}^{3}\right)$, which is the set of functions $f \in$ $L^{2}\left(\omega_{*}, \omega^{*}\right)$ such that the distributional derivative $f^{\prime}$ belongs to $L^{2}\left(\omega_{*}, \omega^{*}\right)$. This space, equipped with the norm

$$
\|f\|_{H^{1}}:=\left(\int_{\omega_{*}}^{\omega^{*}}\left|f^{\prime}(\omega)\right|^{2}+|f(\omega)|^{2} d \omega\right)^{1 / 2}
$$

is a Banach space.

In other words, this system may be considered to be a differential equation of the form

$$
\left\{\begin{array}{l}
\dot{M}(t)=F(t, M(t)) \\
M(0)=M^{0} \in H^{1}\left(\left(\omega_{*}, \omega^{*}\right), \mathbb{S}^{2}\right)
\end{array}\right.
$$

where $F(t, M)$ is a continuous map

$$
F: \mathbb{R} \times H^{1}\left(\left(\omega_{*}, \omega^{*}\right), \mathbb{R}^{3}\right) \rightarrow H^{1}\left(\left(\omega_{*}, \omega^{*}\right), \mathbb{R}^{3}\right) .
$$

3 Or at least they are solutions of the corresponding integral equations.
Moreover, $F$ is periodic in $t$ and locally Lipschitz in $M$. Using the same ideas as in the proof of the CauchyLipschitz (Picard-Lindelöf) theorem, we get the following result, whose proof is detailed in Appendix.

Proposition 1. For every initial condition $M^{0}$ belonging to $H^{1}\left(\left(\omega_{*}, \omega^{*}\right), \mathbb{S}^{2}\right)$, the closed loop system (6), (11) admits a unique solution $M$ in $C^{1}\left([0, \infty), H^{1}\left(\left(\omega_{*}, \omega^{*}\right), \mathbb{S}^{2}\right)\right)$ such that $M(0)=M^{0}$.

\subsection{Closed-loop simulations}

We assume here $\omega_{*}=0, \omega^{*}=1$ and we solve numerically the $T$-periodic system (5) with the $T$-periodic feedback law (11) where $Z=x+\imath y$ and $\Omega=v-\imath u$. The parameters are $T=2 \pi /\left(\omega^{*}-\omega_{*}\right), K_{p}=1, G=\frac{1}{2 T^{2}}$. The simulation is for $t \in\left[0, T_{f}\right], T_{f}=50 T$. The profile $\left[\omega_{*}, \omega^{*}\right] \ni \omega \mapsto(x(t, \omega), y(t, \omega), z(t, \omega))$ is discretized $\{0, \ldots, N\} \ni k \mapsto\left(x_{k}(t), y_{k}(t), z_{k}(t)\right)$ with a regular mesh of step $\epsilon_{N}=\frac{\omega^{*}-\omega_{*}}{N}$ with $N=100$ : $\left(x_{k}(t), y_{k}(t), z_{k}(t)\right)$ is then an approximation of $\left(x\left(t, k \epsilon_{N}\right), y\left(t, k \epsilon_{N}\right), z\left(t, k \epsilon_{N}\right)\right)$. We have checked that the closed-loop simulations are almost identical for $N=100$ and $N=200$. In the feedback law (11), the integral versus $\omega$ is computed assuming that $(x, y, z)$ and $\left(x^{\prime}, y^{\prime}, z^{\prime}\right)$ are constant over ]$\left(k-\frac{1}{2}\right) \epsilon_{N},\left(k+\frac{1}{2}\right) \epsilon_{N}\left[\right.$, their values being $\left(x_{k}, y_{k}, z_{k}\right)$ and $\left(\frac{x_{k+1}-x_{k-1}}{2 \epsilon_{N}}, \frac{y_{k+1}-y_{k-1}}{2 \epsilon_{N}}, \frac{z_{k+1}-z_{k-1}}{2 \epsilon_{N}}\right)$. The obtained differential system is of dimension $3(N+1)$. It is integrated via an explicit Euler scheme with a step size $h=T / 50$. We have tested that $h=T / 100$ yields to almost the same numerical solution at $t=T_{f}=50 T$. After each time-step the new values of $\left(x_{k}, y_{k}, z_{k}\right)$ are normalized to remain in $\mathbb{S}^{2}$.

Figures 1 and 2 summarize the main convergence issues when the initial $\omega$-profiles of $(x, y, z) \in \mathbb{S}^{2}$ are $z(0, \omega)=$ $0.8-0.1 \sin (4 \pi \omega), x(0, \omega)=\cos (\pi \omega) \sqrt{1-z^{2}(0, \omega)}$ and $y(0, \omega)=\sin (\pi \omega) \sqrt{1-z^{2}(0, \omega)}$. The convergence speed is rapid at the beginning and tends to decrease at the end. The control problem is quite hard due to the fact that one has a continuous spectrum, that is, an infinite ensemble of systems with a common control input $\Omega(t)$. Hence, as time increases, the control must fight against the dispersion of the solutions $M(t, \omega)$ for different values of $\omega$. Simulations (not presented here) on much longer times until $10^{4} \mathrm{~T}$ and with the same initial conditions and parameters always yield to smaller final value for the Lyapunov function (for example we get $\left.L\left(10^{4} T\right)=0.0395\right)$. This is a strong indication of asymptotic converge of the profile $M(t, \omega)$ toward $-e_{3}$ even if the convergence speed seems to be very slow. This numerically observed convergence is confirmed by theorem 1 here below.

\section{MAIN RESULT}

The main result of this paper shows that the control law (11) is a solution of the local stabilization problem stated at the end of the introduction.

Theorem 1. There exists $\delta^{\prime}>0$ such that, for every $M_{0} \in H^{1}\left(\left(\omega_{*}, \omega^{*}\right), \mathbb{S}^{2}\right)$ with $\left\|M_{0}+e_{3}\right\|_{H^{1}} \leq \delta^{\prime}$,

$$
M(t) \rightarrow-e_{3} \text { weakly in } H^{1} \text { when } t \rightarrow+\infty \text {. }
$$

In particular, as the injection of $H^{1}$ in $C^{0}$ is compact, $M(t, \omega)$ converges to $-e_{3}$ when $t \rightarrow+\infty$ uniformly with 

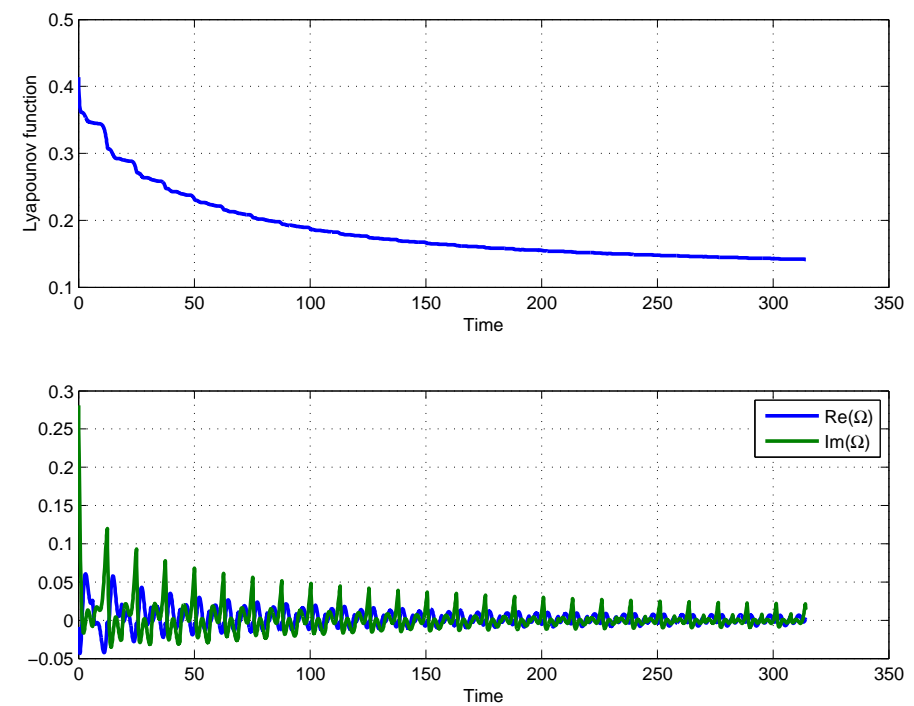

Fig. 1. Lyapunov function $\mathcal{L}(t)$ defined by (8) and the closed-loop control $\Omega(t)$ defined by (11) .
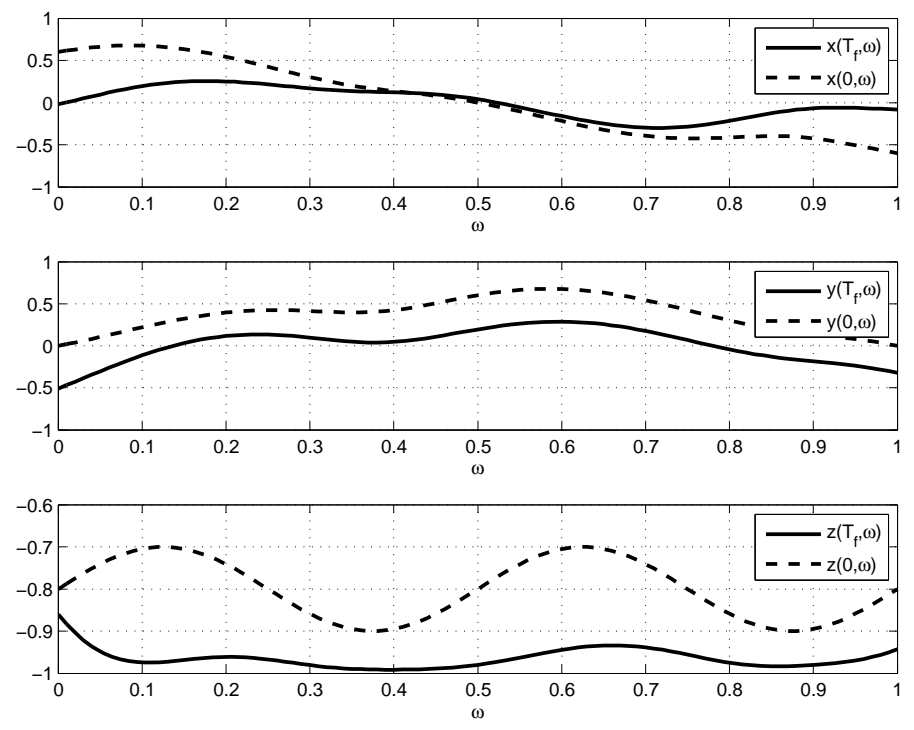

Fig. 2. Initial $(t=0)$ and final $\left(t=T_{f}\right) \omega$-profiles for $x$, $y$ and $z$ solutions of the closed-loop system (5) with the $T$-periodic feedback (11).

respect to $\omega \in\left(\omega_{*}, \omega^{*}\right)$ (convergence in the sup norm of $\left.C^{0}\right)$.

The proof of Theorem 1 relies on an adaptation of the LaSalle invariance principle to infinite dimensional systems. The first step of the proof consists in checking that, locally, the invariant set is reduced to $\left\{-e_{3}\right\}$.

Proposition 2. There exists $\delta>0$ such that, for every $M^{0} \in H^{1}\left(\left(\omega_{*}, \omega^{*}\right), \mathbb{S}^{2}\right)$ with $\left\|M^{0}+e_{3}\right\|_{H^{1}}<\delta$, the map $t \mapsto \mathcal{L}(t)$ is constant on $[0,+\infty)$ if and only if $M^{0}=-e_{3}$.

Proof: We can absorb the positive parameter $G$ via a scaling on $\omega$. Thus we will assume during all the following proofs that $G=1$. Let us assume that $\mathcal{L}(t)$ is constant. Then, $\Omega(t)=0, Z(t, \omega)=Z_{0}(\omega), z(t, \omega)=z_{0}(\omega)$ and we have

$$
\int_{\omega_{*}}^{\omega^{*}}\left\{\imath t\left(Z_{0}^{\prime} z_{0}-Z_{0} z_{0}^{\prime}\right)+Z_{0} z_{0}\right\} e^{\imath \omega t} d \omega=0, \forall t \in[0, T] .
$$

Considering the power series expansion versus $t$ of the left hand side, we get, for any polynomials $P \in \mathbb{C}[\omega]$

$$
\int_{\omega_{*}}^{\omega^{*}}\left[P^{\prime}\left(Z_{0}^{\prime} z_{0}-Z_{0} z_{0}^{\prime}\right)+P Z_{0} z_{0}\right] d \omega=0
$$

Polynomials are dense in $H^{1}$, thus, the previous equality holds for every $P \in H^{1}\left(\omega_{*}, \omega^{*}\right)$. In particular, with $P(\omega)=$ $\overline{Z_{0}(\omega)}$, we get

$$
\int_{\omega_{*}}^{\omega^{*}}\left[\overline{Z_{0}^{\prime}}\left(Z_{0}^{\prime} z_{0}-Z_{0} z_{0}^{\prime}\right)+\overline{Z_{0}} Z_{0} z_{0}\right] d \omega=0
$$

We deduce that

$$
\begin{aligned}
& \int_{\omega_{*}}^{\omega^{*}}\left[\left|Z_{0}^{\prime}\right|^{2}+\left|Z_{0}\right|^{2}\right] d \omega= \\
& \int_{\omega_{*}}^{\omega^{*}}\left[\left(1+z_{0}\right)\left(\left|Z_{0}^{\prime}\right|^{2}+\left|Z_{0}\right|^{2}\right)-\overline{Z_{0}^{\prime}} Z_{0} z_{0}^{\prime}\right] d \omega .
\end{aligned}
$$

This relation provides an inequality of the type

$$
\left\|Z_{0}\right\|_{H^{1}}^{2} \leq C\left(M_{0}\right)\left\|Z_{0}\right\|_{H^{1}}^{2}
$$

where the constant $C\left(M_{0}\right)$ tends to 0 when $M_{0}$ tends to $-e_{3}$ in $H^{1}$. There exists $\delta>0$ such that, for every $M_{0} \in$ $H^{1}\left(\left(\omega_{*}, \omega^{*}\right), \mathbb{S}^{2}\right)$ with $\left\|M_{0}+e_{3}\right\|_{H^{1}}<\delta$, we have $C\left(M_{0}\right)<$ 1. If $\mathcal{L}$ is constant along the trajectory associated to such an initial condition $M^{0}$, then, the previous argument shows that $Z_{0}=0$, thus $M^{0}=-e_{3}$.

Remark 1. The relation (12) shows that the invariant set contains at least $-e_{3}, e_{3}$ and any function $M^{0}$ taking values in the equator (i.e. for which $z_{0}=0$ ). Thus, with these feedback laws, global stabilization (i.e. for every $\left.M^{0} \in H^{1}\left(\left(\omega_{*}, \omega^{*}\right), \mathbb{S}^{2}\right)\right)$ cannot be expected. In order to get global stabilization results, one needs other tools.

For the proof of Theorem 1, we need the continuity with respect to initial conditions, of the solutions of the closed loop system $(6),(11)$, for the $H^{\frac{1}{2}}\left(\omega_{*}, \omega^{*}\right)$-topology. This space is defined by interpolation between $L^{2}\left(\omega_{*}, \omega^{*}\right)$ and $H^{1}\left(\omega_{*}, \omega^{*}\right)$ and we have a compact injection $H^{1}\left(\omega_{*}, \omega^{*}\right) \rightarrow$ $H^{\frac{1}{2}}\left(\omega_{*}, \omega^{*}\right)$. First, let us recall the following Lemma.

Lemma 1. Let $T>0$. There exists $c_{1}>0$ such that, for every $\varphi \in H^{\frac{1}{2}}\left(\omega_{*}, \omega^{*}\right)$ and for every $\alpha \in[0, T]$, the map $\omega \mapsto \varphi(\omega) e^{\imath \alpha \omega}$ belongs to $H^{\frac{1}{2}}\left(\omega_{*}, \omega^{*}\right)$ and satisfies

$$
\left\|\varphi(\omega) e^{\imath \alpha \omega}\right\|_{H^{\frac{1}{2}}} \leq c_{1}\|\varphi\|_{H^{\frac{1}{2}}}
$$

Proof: We have

$$
\left\|\varphi(\omega) e^{\imath \alpha \omega}\right\|_{L^{2}}=\|\varphi\|_{L^{2}}, \forall \varphi \in L^{2}\left(\omega_{*}, \omega^{*}\right),
$$

and, for every $\varphi \in H^{1}\left(\omega_{*}, \omega^{*}\right)$,

$$
\begin{aligned}
& \left\|\varphi(\omega) e^{\imath \alpha \omega}\right\|_{H^{1}} \\
= & \left(\int_{\omega_{*}}^{\omega^{*}}\left|\varphi^{\prime}(\omega)+i \alpha \varphi(\omega)\right|^{2}+|\varphi(\omega)|^{2} d \omega\right)^{1 / 2} \\
\leq & \left(\int_{\omega_{*}}^{\omega^{*}} 2\left|\varphi^{\prime}(\omega)\right|^{2}+\left(2 \alpha^{2}+1\right)|\varphi(\omega)|^{2} d \omega\right)^{1 / 2}
\end{aligned}
$$

thus we get the conclusion with, for example, $c_{1}:=\left(2 T^{2}+\right.$ $2)^{1 / 4}$ by interpolation. 
Proposition 3. There exists $\delta^{\prime}>0$ such that, for every $\left(M_{n}^{0}\right)_{n \in \mathbb{N}} \in H^{1}\left(\left(\omega_{*}, \omega^{*}\right), \mathbb{S}^{2}\right)^{\mathbb{N}}, M_{\infty}^{0} \in H^{1}\left(\left(\omega_{*}, \omega^{*}\right), \mathbb{S}^{2}\right)$ such that

- $\left\|M_{n}^{0}+e_{3}\right\|_{H^{1}}<\delta^{\prime}, \forall n \in \mathbb{N}$,

- $M_{n}^{0} \rightarrow M_{\infty}^{0}$ weakly in $H^{1}$ when $n \rightarrow+\infty$,

- $M_{n}^{0} \rightarrow M_{\infty}^{0}$ strongly in $H^{\frac{1}{2}}$ when $n \rightarrow+\infty$,

the solutions $M_{n}(t, \omega), M_{\infty}(t, \omega)$ of the closed loop system associated to these initial conditions satisfy the following convergences, when $n \rightarrow+\infty$, for every $t \in[0,+\infty)$, $M_{n}(t) \rightarrow M_{\infty}(t)$ strongly in $H^{\frac{1}{2}}, \Omega_{n}(t) \rightarrow \Omega_{\infty}(t)$.

Proof: First let us emphasize that $\sqrt{\mathcal{L}(M)}$ and $\| M+$ $e_{3} \|_{H^{1}}$ are equivalent norms on a small enough $H^{1}\left(\left(\omega_{*}, \omega^{*}\right), \mathbb{S}^{2}\right)$ neighborhood of $-e_{3}$ : there exists $\eta, c_{*}, c^{*}>0$ such that, for every $M \in H^{1}\left(\left(\omega_{*}, \omega^{*}\right), \mathbb{S}^{2}\right)$ with $\left\|M^{0}+e_{3}\right\|_{H^{1}}<\eta$, we have

$$
c_{*} \sqrt{\mathcal{L}(M)} \leq\left\|M+e_{3}\right\|_{H^{1}} \leq c^{*} \sqrt{\mathcal{L}(M)} .
$$

Indeed, we have

$$
\left\|M+e_{3}\right\|_{H^{1}}^{2}=\int_{\omega_{*}}^{\omega^{*}}\left(\left|Z^{\prime}\right|^{2}+\left(z^{\prime}\right)^{2}+|Z|^{2}+(1+z)^{2}\right) d \omega .
$$

When $M \in H^{1}\left(\left(\omega_{*}, \omega^{*}\right), \mathbb{S}^{2}\right)$ is close enough to $-e_{3}$ in $H^{1}$, we have $\|Z\|_{L^{\infty}}<1 / \sqrt{2}$ (because the injection $H^{1} \rightarrow L^{\infty}$ is continuous), which implies

$$
\frac{1}{2 \sqrt{2}}|Z|^{2} \leq 1+z=1-\sqrt{1-|Z|^{2}} \leq \frac{1}{2}|Z|^{2} .
$$

Now, let $\delta^{\prime}:=\min \left\{\delta c_{*} / c^{*}, \eta\right\}$, where $\delta$ is as in Proposition 2. Thanks to the monotonicity of $\mathcal{L}$, we have, for every $t \in[0,+\infty)$,

$$
\begin{aligned}
\left\|M_{n}(t)+e_{3}\right\|_{H^{1}} \leq c^{*} \sqrt{\mathcal{L}\left(M_{n}(t)\right)} \leq c^{*} \sqrt{\mathcal{L}\left(M_{n}^{0}\right)} \\
\leq \frac{c^{*}}{c_{*}}\left\|M_{n}^{0}+e_{3}\right\|_{H^{1}}<\frac{c^{*} \delta^{\prime}}{c_{*}} \leq \delta .
\end{aligned}
$$

We have

$$
\begin{aligned}
\| M_{n}(t)- & M_{\infty}(t)\left\|_{H^{\frac{1}{2}}} \leq\right\| M_{n}^{0}-M_{\infty}^{0} \|_{H^{\frac{1}{2}}} \\
& +\int_{0}^{t}\left\|F\left(s, M_{n}(s)\right)-F\left(s, M_{\infty}(s)\right)\right\|_{H^{\frac{1}{2}}} d s .
\end{aligned}
$$

Let us prove the existence of $C>0$ such that, for every $M, \tilde{M} \in H^{1}\left(\omega_{*}, \omega^{*}\right)$ satisfying $\left\|M+e_{3}\right\|_{H^{1}}<\delta$, we have

$$
\|F(s, M)-F(s, \tilde{M})\|_{H^{\frac{1}{2}}} \leq C\|M-\tilde{M}\|_{H^{\frac{1}{2}}}, \forall s \in \mathbb{R} .
$$

Then, the proof may be concluded thanks to the Gronwall Lemma. Let us work, for example, on the third component of $F$ :

$$
F_{3}(t, M)=-R e\left[\Omega(t) \bar{Z} e^{-\imath \omega \int_{0}^{t} \zeta}\right],
$$

where $\Omega$ is defined by (11). We have

$$
\begin{gathered}
\left\|F_{3}(t, M)-F_{3}(t, \tilde{M})\right\|_{H^{\frac{1}{2}}} \leq \\
|\Omega(t)-\tilde{\Omega}(t)|\left\|\bar{Z} e^{-\imath \omega \int_{0}^{t} \zeta}\right\|_{H^{\frac{1}{2}}}+|\tilde{\Omega}(t)|\left\|\overline{(Z-\tilde{Z})} e^{-\imath \omega \int_{0}^{t} \zeta}\right\|_{H^{\frac{1}{2}}} \\
\leq|\Omega(t)-\tilde{\Omega}(t)| c_{1}\|Z\|_{H^{\frac{1}{2}}}+K c_{1}\|Z-\tilde{Z}\|_{H^{\frac{1}{2}}}
\end{gathered}
$$

where $c_{1}$ is as in the previous Lemma and $K=K(\delta)$. It is sufficient to prove the existence of a constant $C>0$ such that, for every $M, \tilde{M} \in H^{1}\left(\left(\omega_{*}, \omega^{*}\right), \mathbb{S}^{2}\right)$ satisfying $\left\|M+e_{3}\right\|_{H^{1}},\left\|\tilde{M}+e_{3}\right\|_{H^{1}}<\delta$, we have

$$
|\Omega(t)-\tilde{\Omega}(t)| \leq C\|M-\tilde{M}\|_{H^{\frac{1}{2}}}, \forall t \in[0,+\infty) .
$$

Let us prove it only on one of the terms that compose $\Omega$ (the other terms may be treated as well):

$$
\begin{aligned}
& \left|\int_{\omega_{*}}^{\omega^{*}}\left(Z^{\prime} z-\tilde{Z}^{\prime} \tilde{z}\right) e^{\imath|\omega| \int_{0}^{t} \zeta} d \omega\right| \leq \\
& \left|\int_{\omega_{*}}^{\omega^{*}}\left(Z^{\prime}-\tilde{Z}^{\prime}\right) z e^{\imath|\omega| \int_{0}^{t} \zeta} d \omega\right|+\left|\int_{\omega_{*}}^{\omega^{*}} \tilde{Z}^{\prime}(z-\tilde{z}) e^{\imath|\omega| \int_{0}^{t} \zeta} d \omega\right| \\
\leq & \left\|Z^{\prime}-\tilde{Z}^{\prime}\right\|_{H^{-\frac{1}{2}}}\left\|z e^{\imath|\omega| \int_{0}^{t} \zeta}\right\|_{H^{\frac{1}{2}}}+\left\|\tilde{Z}^{\prime}\right\|_{H^{-\frac{1}{2}}}\left\|(z-\tilde{z}) e^{\imath|\omega| \int_{0}^{t} \zeta}\right\|_{H^{\frac{1}{2}}} \\
& \leq c_{1} K\|M-\tilde{M}\|_{H^{\frac{1}{2}}} \cdot
\end{aligned}
$$

Proof of Theorem 1: Let $\delta^{\prime}$ be as in the previous proof. Let $M^{0} \in H^{1}\left(\left(\omega_{*}, \omega^{*}\right), \mathbb{S}^{2}\right)$ be such that $\left\|M^{0}+e_{3}\right\|_{H^{1}}<\delta^{\prime}$ and $M \in C^{1}\left([0,+\infty), H^{1}\left(\left(\omega_{*}, \omega^{*}\right), \mathbb{S}^{2}\right)\right)$ be the solution of the closed loop system such that $M(0)=M^{0}$.

First step: Let us prove that $\Omega(t) \rightarrow 0$ when $t \rightarrow+\infty$.

Thanks to the choice of the feedback law, $M(t)$ is bounded in $H^{1}$, uniformly with respect to $t \in[0,+\infty)$. Computing explicitly $\frac{d \Omega}{d t}(t)$, we see that $\frac{d \Omega}{d t}(t)$ is bounded in $\mathbb{C}$ uniformly with respect to $t \in[0,+\infty)-\mathbb{N} T$. Thus, $\Omega$ is uniformly continuous on $[0,+\infty)$. Since $\Omega \in L^{2}(0,+\infty)$, it has to satisfy $\Omega(t) \rightarrow 0$ (Barbalat's lemma).

Second step: Let us prove that $-e_{3}$ is the only possible weak $H^{1}$ limit. Let $M_{\infty}^{0}$ be a weak $H^{1}$ limit of the trajectory starting from $M^{0}$. There exists a sequence $\left(t_{n}\right)_{n \in \mathbb{N}}$ of $[0,+\infty)$ such that $t_{n} \rightarrow+\infty$,

$$
\begin{aligned}
& M\left(t_{n}\right) \rightarrow M_{\infty}^{0} \text { weakly in } H^{1} \text { when } n \rightarrow+\infty, \\
& M\left(t_{n}\right) \rightarrow M_{\infty}^{0} \text { strongly in } H^{1 / 2} \text { when } n \rightarrow+\infty .
\end{aligned}
$$

Working as in the previous proof, one may prove that

$$
\left\|M\left(t_{n}\right)+e_{3}\right\|_{H^{1}}<\delta, \forall n \in \mathbb{N},
$$

There exists $t_{\infty} \in[0, T)$ such that $t_{n} \bmod T \rightarrow t_{\infty}$. Let $M_{\infty}(t, \omega)$ be the solution of the closed loop system associated to the initial condition $M_{\infty}\left(t_{\infty}\right)=M_{\infty}^{0}$. Let us prove that $\mathcal{L}$ is constant along this trajectory, by proving that the associated control $\Omega_{\infty}$ vanishes. In order to simplify, we assume that $t_{\infty}=0$ (otherwise, consider an additional shift). For every $t>0, M\left(t_{n}+t\right) \rightarrow M_{\infty}(t)$ strongly in $H^{1 / 2}$ when $n \rightarrow+\infty$, thanks to the previous proposition. This allows to pass to the limit in the feedback law: $\Omega\left(t_{n}+t\right) \rightarrow \Omega_{\infty}(t)$ when $n \rightarrow+\infty$, for every $t>0$. Thanks to the first step, we get $\Omega_{\infty}=0$.

In order to apply Proposition 2, we only need to check that $\left\|M_{\infty}^{0}+e_{3}\right\|_{H^{1}}<\delta$, which is a consequence of (14).

\section{CONCLUSION}

We have investigated here the stabilization of an infinite dimensional system admitting in open-loop a continuous spectrum. We have designed a Lyapunov based feedback. Closed-loop simulations illustrate the asymptotic convergence towards the goal steady-state. We have provided a local and weak convergence result for the $H^{1}$ topology. 
Simulations indicate that the domain of attraction is far from being local and thus we can expect a large attraction domain for this feedback law.

More generally, this feedback and convergence analysis open the way to asymptotic stabilization of neutrally stable systems of infinite dimension with continuous spectra. For example, it will be interesting to see if the following system (1D Maxwell-Lorentz model for the propagation of an electro-magnetic wave in a non-homogenous dispersive material)

$$
\begin{aligned}
\frac{\partial^{2} E}{\partial t^{2}}+\frac{\partial^{2} P}{\partial t^{2}}=\frac{\partial^{2} E}{\partial x^{2}}, & \frac{\partial^{2} P}{\partial t^{2}}=p^{2}(x)(E-P), \quad x \in(0,1) \\
E(0, t) & =u(t), \quad E(1, t)=v(t)
\end{aligned}
$$

with two controls $u$ and $v$, can also be stabilized to zero. Notice that when $p(x)$ is a smooth strictly increasing positive functions, this system admits as continuous spectrum $\pm \imath] p(0), p(1)[$.

\section{REFERENCES}

K. Beauchard and J.-M. Coron. Controllability of a quantum particle in a moving potential well. $J$. of Functional Analysis, 232:328-389, 2006.

K. Beauchard, J.-M. Coron, and P. Rouchon. Controllability issues for continuous spectrum systems and ensemble controllability of bloch equations. Communications in Mathematical Physics, volume 296, Number 2, June 2010, p.525-557.

T. Chambrion, P. Mason, M. Sigalotti, and M. Boscain. Controllability of the discrete-spectrum schrödinger equation driven by an external field. Ann. Inst. $H$. Poincaré Anal. Non Linéaire, 26(1):329-349, 2009.

J.M. Coron. Control and Nonlinearity. American Mathematical Society, 2007.

J.S. Li and N. Khaneja. Control of inhomogeneous quantum ensembles. Phys. Rev. A., 73:030302, 2006.

J.S. Li and N. Khaneja. Control of inhomogeneous quantum ensembles. IEEE Trans. Automatic Control, 54(3): 528-536, 2009.

M. Mirrahimi. Lyapunov control of a quantum particle in a decaying potential. Annales de lInstitut Henri Poincaré (c) Nonlinear Analysis, 26:1743-1765, 2009.

V. Nersesyan. Growth of Sobolev norms and controllability of Schrödinger equations. Comm. Math. Phys. (in press), 2010.

\section{Appendix A. PROOF OF PROPOSITION 1}

Let $M^{0} \in H^{1}\left(\left(\omega_{*}, \omega^{*}\right), \mathbb{S}^{2}\right)$ and $R>0$ be such that

$$
R>\left\|M^{0}\right\|_{H^{1}}, \quad R^{2}>\max \{1 / G, 1\}\left(2 \mathcal{L}(0)+\omega^{*}-\omega_{*}\right) .
$$

Let $C_{1}, C_{2}>0$ be such that

$$
\begin{gathered}
\left\|f e^{-\imath \omega t}\right\|_{H^{1}} \leq C_{1}\|f\|_{H^{1}}, \quad \forall f \in H^{1}\left(\omega_{*}, \omega^{*}\right), \forall t \in[0, T], \\
\left\|F\left(t, M_{1}\right)-F\left(t, M_{2}\right)\right\|_{H^{1}} \leq C_{2}\left\|M_{1}-M_{2}\right\|_{H^{1}}, \\
\forall M_{1}, M_{2} \in B_{R}\left[H^{1}\left(\left(\omega_{*}, \omega^{*}\right), \mathbb{R}^{3}\right)\right], \forall t \in[0, T],
\end{gathered}
$$

where $B_{R}[F]$ denote the closed ball centered at 0 with radius $R$, of the space $F$. Let $T^{*}=T^{*}(R)>0$ be small enough so that

$$
\left\|M^{0}\right\|_{H^{1}}+T^{*} C_{1} K_{p}(2 G+1) R^{3}<R \text { and } T^{*} C_{2}<1 \text {. }
$$

Let us consider the map $\Theta$, defined on the space

$$
E:=B_{R}\left[C^{0}\left(\left[0, T^{*}\right], H^{1}\left(\left(\omega_{*}, \omega^{*}\right), \mathbb{R}^{3}\right)\right)\right]
$$

by

$$
\Theta(M)(t, \omega):=M^{0}(\omega)+\int_{0}^{t} F(s, M(s, \omega)) d s
$$

for every $(t, \omega) \in\left[0, T^{*}\right] \times\left(\omega_{*}, \omega^{*}\right)$.

First step: Let us prove that $\Theta$ takes values in E. Let $M \in E$. It is clear that $\Theta(M)$ is continuous in time with values in $H^{1}\left(\left(\omega_{*}, \omega^{*}\right), \mathbb{R}^{3}\right)$. For $t \in\left[0, T^{*}\right]$, we have

$$
\|\Theta(M)(t)\|_{H^{1}} \leq\left\|M^{0}\right\|_{H^{1}}+\int_{0}^{t}\|F(s, M(s))\|_{H^{1}} d s .
$$

By definition, we have

$$
\begin{gathered}
\|F(s, M(s))\|_{H^{1}}^{2}= \\
\left\|\Omega(s) z(s) e^{-\imath \omega \int_{0}^{s} \varsigma}\right\|_{H^{1}}^{2}+\left\|\Re\left[\Omega(s) Z(s) e^{-\imath \omega \int_{0}^{s} \varsigma}\right]\right\|_{H^{1}}^{2} \\
\leq|\Omega(s)|^{2} C_{1}^{2}\left(\|z(s)\|_{H^{1}}^{2}+\|Z(s)\|_{H^{1}}^{2}\right)=C_{1}^{2}|\Omega(s)|^{2}\|M(s)\|_{H^{1}}^{2} .
\end{gathered}
$$

Moreover, the Cauchy-Schwarz inequality gives

thus,

$$
|\Omega(s)| \leq K_{p}(2 G+1)\|M(s)\|_{H^{1}}^{2},
$$

$\|\Theta(M)\|_{L^{\infty}\left(\left(0, T^{*}\right), H^{1}\right)} \leq\left\|M^{0}\right\|_{H^{1}}+T^{*} C_{1} K_{p}(2 G+1) R^{3} \leq R$

thanks to (A.4).

Second step: Let is prove that $\Theta$ is a contraction. For $M_{1}, M_{2} \in E$ and $t \in\left[0, T^{*}\right]$, using (A.3), we get

$$
\begin{aligned}
\left\|\Theta\left(M_{1}\right)(t)-\Theta\left(M_{2}\right)(t)\right\|_{H^{1}} \leq & \int_{0}^{t}\left\|F\left(s, M_{1}(s)\right)-F\left(s, M_{2}(s)\right)\right\|_{H^{1}} \\
& \leq t C_{2}\left\|M_{1}-M_{2}\right\|_{L^{\infty}\left(\left(0, T^{*}\right), H^{1}\right)},
\end{aligned}
$$

thus $\Theta$ is a contraction, thanks to (A.4).

Third step: Let us prove the existence and uniqueness of strong solutions, defined on $[0,+\infty)$. Thanks to the Banach fixed point theorem, the map $\Theta$ has a unique fixed point.

We have proved that, for every $R>0$, there exists $T^{*}=T^{*}(R)>0$ such that, for every $M^{0} \in$ $B_{R}\left[H^{1}\left(\left(\omega_{*}, \omega^{*}\right), \mathbb{S}^{2}\right)\right]$, there exists a unique weak solution

$$
M \in C^{0}\left(\left[0, T^{*}\right], H^{1}\left(\left(\omega_{*}, \omega^{*}\right), \mathbb{R}^{3}\right)\right.
$$

in the sense $M(t, \omega)=M^{0}(\omega)+\int_{0}^{t} F(s, M(s, \omega)) d s$ in $H^{1}\left(\omega_{*}, \omega^{*}\right), \forall t \in\left[0, T^{*}\right]$. We deduce that $M \in$ $C^{1}\left(\left[0, T^{*}\right], H^{1}\left(\left(\omega_{*}, \omega^{*}\right), \mathbb{R}^{3}\right)\right)$ and $\frac{d M}{d t}(t, \omega)=F(t, M(t, \omega))$ in $H^{1}\left(\left(\omega_{*}, \omega^{*}\right), \mathbb{R}^{3}\right), \forall t \in\left[0, T^{*}\right]$. Since the embedding $H^{1} \rightarrow L^{\infty}$ is continuous, we also have $\frac{d M}{d t}(t, \omega)=$ $F(t, M(t, \omega)), \forall(t, \omega) \in\left[0, T^{*}\right] \times\left(\omega_{*}, \omega^{*}\right)$. This has 2 consequences. Firstly, $M(t,$.$) takes values in \mathbb{S}^{2}$ for every $t \in\left[0, T^{*}\right]$, indeed, $M^{0}$ does and the following computation is licit $\frac{d}{d t}\|M(t, \omega)\|^{2}=2\langle M(t, \omega), F(t, M(t, \omega))\rangle=0$. Secondly, the computations $(9),(10)$ are licit, thus $\mathcal{L}(t)$ is not increasing. Therefore, we have

$$
\begin{aligned}
\left\|M\left(T^{*}\right)\right\|_{H^{1}}^{2} & =\frac{1}{2} \int_{\omega_{*}}^{\omega^{*}}\left|Z^{\prime}(T)\right|^{2}+z^{\prime}(T)^{2}+|Z(T)|^{2}+z(T)^{2} d \omega \\
\leq & \max \{1 / G, 1\}\left(2 \mathcal{L}(T)+\omega^{*}-\omega_{*}\right) \\
& \leq \max \{1 / G, 1\}\left(2 \mathcal{L}(0)+\omega^{*}-\omega_{*}\right) \leq R^{2}
\end{aligned}
$$

thanks to (A.1). Thus, we can apply the previous result with $M^{0}$ replaced by $M\left(T^{*}\right)$ : it provides a solution on $\left[0,2 T^{*}\right]$. Iterating this again, we get a solution defined for every $t \in[0,+\infty)$. 